\title{
MULTISCALE SYNTHESIS AND ANALYSIS OF FRACTAL RENEWAL PROCESSES
}

\author{
Warren M. Lam and Gregory W. Wornell \\ Research Laboratory of Electronics \\ Massachusetts Institute of Technology \\ Cambridge, MA 02139
}

\begin{abstract}
A novel multiscale framework is introduced for the representation of a class of fractal point processes. Using this framework, efficient algorithms are developed for the synthesis of fractal point processes from a mixture of Poisson processes. Multiscale analysis algorithms are also developed within this framework for computing Maximum-Likelihood fractal dimension estimates of such processes from corrupted observations.
\end{abstract}

\section{INTRODUCTION}

Point processes with fractal characteristics are promising models for a wide range of natural and man-made phenomena, including distributions of stars and planets in the universe, transmission errors in many communication channels, and impulsive spikes in auditory neural signals [1] [2] [3] [4]. In contrast to fractal waveforms, which have been explored in considerable depth (see, e.g., [5]), the development of efficient algorithms for synthesizing, analyzing, and processing fractal point processes has generally proven difficult, largely due to the lack of an adequate mathematical framework. In this paper, we present a novel and rather natural multiscale framework for the study of an important class of fractal point processes, and describe some practical and efficient signal processing algorithms that arise out of this framework.

Before we present our main results, we briefly summarize some terminology and notation for the paper. In general, a point process refers to a collection of points, typically called "arrivals," that are randomly distributed over some multidimensional space. For simplicity, we shall restrict our discussion to the one-dimensional case and refer to the underlying space as time $t$. For convenience, we will also choose the time origin to coincide with an arrival referred to as the zeroth arrival, and consider only $t \geq 0$. In this case it is useful to characterize a point process in terms of the collection of time intervals between arrivals. In particular, we use $X[n]$ for $n=1,2, \ldots$ to denote the $n$th interarrival time-specifically, the time interval between the $(n-1)$ st and $n$th arrivals. An equivalent characterization of a point process is in terms of $N_{X}(t)$, the total number of arrivals

This work has been supported in part by the Advanced Research Projects Agency monitored by ONR under Contract No. N00014-93-1-0686, and the Air Force Office of Scientific Research under Grant No. AFOSR-91-0034. that have occurred up to and including time $t$. The counting process $N_{X}(t)$ is a discrete-valued continuous-time random process whose generalized derivative consists of a train of unit impulses located at the arrival instants.

\section{A FRACTAL POINT PROCESS MODEL}

The point processes of interest in this work are those that possess a key self-similarity property. Formally, a self-similar point process is defined to be a point process that is statistically scale-invariant in the strict sense, so that the associated counting process $N_{X}(t)$ obeys, for all $a>0$,

$$
N_{X}(t) \stackrel{p}{=} N_{X}(a t),
$$

where the notation $\stackrel{p}{=}$ denotes equality in the sense of all finite-dimensional distributions.

Many physical phenomena of interest exhibit no preference for a space or time origin. Consequently, we are generally interested in point process models that are characterized by some form of stationarity. Since renewal processesi.e., processes with independent, identically-distributed interarrivals - are widely used to generate stationary point process models, it is tempting to restrict our attention to those self-similar point processes that are simultaneously renewal processes. However, it is straightforward to show no nontrivial self-similar point processes are bona fide renewal processes (see, e.g., [6]).

Fortunately, a weaker but still highly meaningful form of stationarity can be imposed by generalizing the notion of a renewal process. This notion is based on a characterization of the point process after subcollections of interarrival intervals are discarded. Specifically, we say a point process is conditionally-renewing if it has the following properties:

1. When interarrivals not in the range $(\underline{x}, \bar{x}]$ are discarded, for some $0<\underline{x}<\bar{x}<\infty$, the resulting process is a renewal process; and

2. Any finite collection of point processes, the ith process of which is derived by removing interarrivals not in some range $\left(\underline{x}_{i}, \bar{x}_{i}\right]$ for some $0<\underline{x}_{i}<\bar{x}_{i}<\infty$, are mutually independent when the ranges $\left(\underline{x}_{i}, \bar{x}_{i}\right]$ for distinct $i$ are nonoverlapping.

It is insightful to note that the conditioning in this definition is physically rather natural. Indeed, in empirical tests for renewal behavior in many physical point processes, limitations on data resolution and duration typically preclude the 
measurements of very short and very long interarrivals. It is also worth noting that our definition is closely related to the concept of conditional stationarity developed by Mandelbrot in [2].

In the sequel, we restrict our attention to those selfsimilar point processes which are conditionally-renewing. For convenience, we shall refer to this class of processes as simply fractal renewal processes. From this definition, it can be shown [6] that these processes have the key property that upon removal of the interarrivals not in the range $\left(x_{L}, x_{H}\right]$, where $x_{L}$ and $x_{H}$ are any constants such that $0<x_{L}<x_{H}<\infty$, the resulting renewal process has interarrivals $Y[n]$ distributed according to the probability density function

$$
f_{Y}(y)= \begin{cases}\sigma^{2} / y^{\gamma} & x_{L}<y \leq x_{H} \\ 0 & \text { otherwise, }\end{cases}
$$

where $\sigma^{2}$ is a normalization constant. The shape parameter $\gamma$ of this power-law distribution often lies between 1 and 2 , and is related to the fractal dimension $D$ of the point process via

$$
\gamma=D+1 \text {. }
$$

\section{A SYNTHESIS ALGORITHM FOR FRACTAL RENEWAL PROCESSES}

In this section, we develop a multiscale synthesis for fractal renewal processes. This synthesis involves the mixture of a continuum collection of constituent processes indexed by a real variable $a \in[\underline{a}, \bar{a}]$. These constituents are obtained from different dilations of independent sample functions derived from a prototype Poisson process with mean arrival rate $\lambda$. Specifically, the interarrivals $W_{a}[n]$ of each constituent are related to the interarrivals $W[n]$ of the prototype by

$$
W_{a}[n] \stackrel{p}{=} e^{a} W[n]
$$

Since the amount of expansion increases with $a$, the real constants $\underline{a}$ and $\bar{a}$ can be interpreted as the indices of the finest and coarsest scales, respectively.

Using this family of Poisson processes, a point process is generated as follows. The synthesis is initialized by locating the zeroth arrival of the output at the origin. For the generation of each subsequent arrival, a constituent process is first selected independently from a generalized exponential probability density function

$$
f_{A}(a)= \begin{cases}\sigma_{A}^{2} \exp [-(\gamma-1) a] & \underline{a} \leq a \leq \bar{a} \\ 0 & \text { otherwise, }\end{cases}
$$

where $\sigma_{A}^{2}$ is a normalization factor, and $\gamma$ is a free parameter. The $n$th arrival of the output is then set at the first arrival time of the selected constituent process following the $(n-1)$ st arrival time of the output.

In [6] we show that the point process generated in this manner is a renewal process with the property that, as $\underline{a} \rightarrow$ $-\infty$ and $\bar{a} \rightarrow \infty$, the probability density function of its interarrivals $X[n]$ is a power-law, i.e.,

$$
\frac{f_{X}(x)}{\sigma_{A}^{2}} \rightarrow \frac{\sigma_{0}^{2}}{x^{\gamma}}
$$

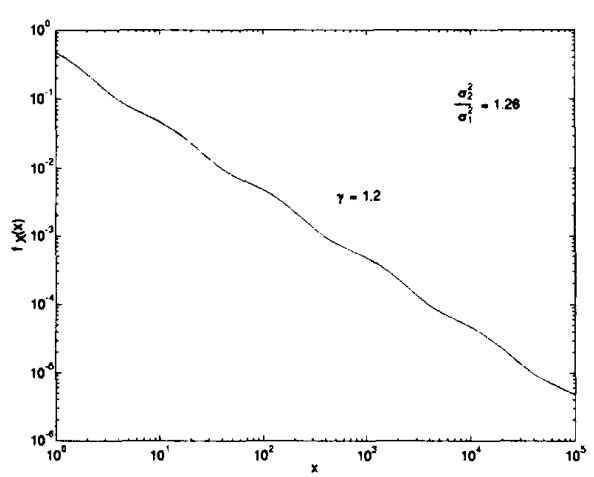

Figure 1: Interrarival density, discrete synthesis.

for $x>0$, where

$$
\sigma_{0}^{2}=\frac{\Gamma(\gamma-1)}{\lambda^{\gamma-1}}
$$

More generally, we note that (3) with (4) is a good approximation to the interarrival density for values of $x$ satisfying

$$
e^{a} / \lambda \ll x \ll e^{\bar{a}} / \lambda
$$

Consequently, the values of $\underline{a}$ and $\bar{a}$ required in practice depend on the interarrival range of interest.

Very useful approximations to fractal renewal process behavior are obtained when the continuum of constituents in the preceding synthesis is replaced with a discrete collection of constituents, which we index using the integer variable $m \in\{\underline{m}, \underline{m}+1, \ldots, \bar{m}\}$. In this case, the interarrivals $W_{m}[n]$ of each of these constituents are related to the interarrivals $W[n]$ of the prototype via

$$
W_{m}[n] \stackrel{\mathcal{P}}{=} \rho^{m} W[n],
$$

where the constant $\rho>1$ governs the spacing between the constituents. The integers $\underline{m}$ and $\bar{m}$ can then be regarded as the indices of the finest and coarsest scales, respectively. The mixing of the constituents is carried out as in the continuum case, but with the integer-valued selection random variables $M_{n}$ distributed according to the generalized geometric probability mass function

$$
\mathrm{P}\{M=m\}= \begin{cases}\sigma_{M}^{2} \rho^{-(\gamma-1) m} & m=\underline{m}, \underline{m}+1, \ldots, \bar{m} \\ 0 & \text { otherwise, }\end{cases}
$$

where $\sigma_{M}^{2}$ is a nornalization factor.

As shown in [6], the process synthesized in this way is a renewal process with the property that, as $\underline{m} \rightarrow-\infty$ and $\bar{m} \rightarrow \infty$, its interarrival density satisfies

$$
\frac{\sigma_{1}^{2}}{x^{\gamma}} \leq \frac{f_{X}(x)}{\sigma_{M}^{2}} \leq \frac{\sigma_{2}^{2}}{x^{\gamma}}
$$

for some constants $0<\sigma_{1}^{2} \leq \sigma_{2}^{2}<\infty$, and for every $x>0$. Again, we stress that the number of scales required in practice depends on the interarrival range of interest. Fig. 1 
shows the interarrival density corresponding to the case $\rho=10$ and $\gamma=1.2$. As one would anticipate, the ripple periodicity is $\log \rho$. Furthermore, it is worth noting that ripple size decreases rapidly as $\rho \rightarrow 1$, leading to increasingly fine approximations. When $\rho=2$, for example, the approximation is essentially perfect; indeed, numerical calculations yield $\log \left(\sigma_{2}^{2} / \sigma_{1}^{2}\right)=3.2 e-5$ for the case $\gamma=1.2$ ! Not surprisingly, decreasing $\rho$ involves a tradeoff-finer approximations at the expense of requiring more constituents for a given interarrival range of interest.

As a final remark, we note that a statistically equivalent but more efficient implementation of the multiscale synthesis arises by exploiting the memoryless property of Poisson processes. In particular, we can generate a fractal renewal process from just a single prototype Poisson process. With this method, the nth interarrival interval of the Poisson process is stretched by the factor $\rho^{m_{n}}$, where $m_{n}$ is the value of the $n$th selection random variable. From this point of view, there are interesting connections between our construction and the construction of Johnson, et al.[7] based on nonhomogeneous Poisson processes.

\section{A PARAMETER ESTIMATION ALGORITHM FOR FRACTAL RENEWAL PROCESSES}

In this section, we demonstrate how the discrete multiscale representation developed in Section 3 can be exploited in the estimation of the shape parameter $\gamma$ associated with a fractal renewal process. As discussed in Section 2, this parameter is directly related to the fractal dimension of the process and in general captures useful information about the pattern of arrivals in the associated point process. In addition, estimates of $\gamma$ are required in intermediate stages of many detection and estimation problems involving such processes.

In the sequel, we present a Maximum-Likelihood (ML) algorithm for estimating $\gamma$ based on observations of interarrivals. For robustness, the observations will be modeled as distorted. In particular, we assume observations of the form

$$
Y[n]=X[n]+W[n], \quad n=1,2, \ldots, N,
$$

where $\{X[n] ; n=1,2, \ldots, N\}$ are the interarrivals of a fractal renewal process and $\{W[n] ; n=1,2, \ldots, N\}$ are "noise" terms. We restrict our attention to the case in which the $W[n]$ are both mutually independent and independent of the interarrivals $X[n]$, and are identically distributed according to the probability density function

$$
f_{W}(w)= \begin{cases}\alpha \exp (-\alpha w) & w \geq 0 \\ 0 & \text { otherwise }\end{cases}
$$

This noise component can be used for modeling a variety of natural effects that arise in applications, such as a random processing delay in an interarrival measurement transducer.

For convenience, we formulate our problem in terms of a new parameter $\beta$ defined as

$$
\beta=\rho^{1-\gamma},
$$

keeping in mind that the ML estimate of $\gamma$ can be obtained from the resulting $M L$ estimate of $\beta$ via

$$
\hat{\gamma}_{\mathrm{ML}}=1-\log \hat{\beta}_{\mathrm{ML}} / \log \rho .
$$

In addition to $\gamma$, the parameters $\lambda$ and $\alpha$ are generally unknown a priori, and need to be estimated. Consequently, we represent the collection of parameters to be jointly estimated with the vector $\Theta=(\lambda, \alpha, \beta)^{T}$. We also note that without loss of generality we may set $\underline{m}=1$ (and appropriately scale $\lambda$ ). The total number of scales required, which we denote by $L$, is typically determined from the spread of the data. As will become apparent, overestimating $L$ generally does not affect the estimation performance, though the corresponding algorithm is less efficient in terms of both computation and storage.

Direct calculation of the ML parameter estimates is difficult in general. However, these estimates can be efficiently computed using an iterative Estimate-Maximize (EM) algorithm [8]. In our description of the algorithm, we use $\hat{\Theta}_{[r]}=\left(\hat{\lambda}_{[r]}, \hat{\alpha}_{[r]}, \hat{\beta}_{[r]}\right)^{T}$ to denote the estimates obtained at iteration $r$, and, for convenience, $\hat{\lambda}_{m[r]}$ to denote $\hat{\lambda}_{[r]} \rho^{-m}$. Each iteration of the resulting estimation algorithm consists of two steps.

E-Step. Using the current set of parameter estimates, for each $m$ and $n$ estimate the probability that interarrival $x[n]$ was derived from scale $m$ given the observation $y[n]$, i.e.,

$$
\begin{aligned}
P\left(m \mid y[n] ; \hat{\Theta}_{[r]}\right)= & \sigma_{i}^{2} \frac{1-\hat{\beta}_{[r]}}{1-\hat{\beta}_{[r]}^{L}} \hat{\beta}_{[r]}^{m-1} \frac{\hat{\lambda}_{m[r]} \hat{\alpha}_{[r]}}{\hat{\lambda}_{m[r]}-\hat{\alpha}_{[r]}} \times \\
& {\left[\exp \left(-\hat{\alpha}_{[r]} y[n]\right)-\exp \left(-\hat{\lambda}_{m[r]} y[n]\right)\right] }
\end{aligned}
$$

where $\sigma_{i}^{2}$ is a normalization constant and provided $\hat{\lambda}_{m[r]} \neq$ $\hat{\alpha}_{[r]}$. When $\hat{\lambda}_{m[r]}=\hat{\alpha}_{[r]}$, we use the alternative expression

$P\left(m \mid y[n] ; \hat{\Theta}_{[r]}\right)=\tilde{\sigma}_{i}^{2} \frac{1-\hat{\beta}_{[r]}}{1-\hat{\beta}_{[r]}^{L}} \hat{\beta}_{[r]}^{m-1} \hat{\lambda}_{m[r]}^{2} y[n] \exp \left(-\hat{\lambda}_{m[r]} y[n]\right)$

where $\tilde{\sigma}_{i}^{2}$ is again a normalization constant.

M-Step. Using the preceding table of probability estimates, new estimates of the parameters are computed via

$$
\begin{aligned}
& \frac{1}{\hat{\lambda}_{[r+1]}}= \frac{1}{N} \sum_{n=1}^{N} \sum_{m=1}^{L} P\left(m \mid y[n] ; \hat{\Theta}_{[r]}\right) \rho^{-m} \times \\
& E\left[x[n] \mid y[n], m ; \hat{\Theta}_{[r]}\right] \\
& \frac{1}{\hat{\alpha}_{[r+1]}}= \frac{1}{N} \sum_{n=1}^{N} \sum_{m=1}^{L} P\left(m \mid y[n] ; \hat{\Theta}_{[r]}\right) \times \\
& \frac{1}{1-\hat{\beta}_{[r+1]}}=\left.\frac{1}{N} \sum_{n=1}^{N} \sum_{m=1}^{L} P(m \mid n]-E\left[x[n] \mid y[n], m ; \hat{\Theta}_{[r]}\right]\right) \\
& \text { where } \\
& E[x[n] \mid y[n], m ; \hat{\Theta}]\left.=\frac{1}{\hat{\lambda}_{m}-\hat{\alpha}}-\frac{\exp \left(\left(\hat{\lambda}_{m}-\hat{\alpha}\right) y[n]\right)-1}{y[n]}\right)
\end{aligned}
$$




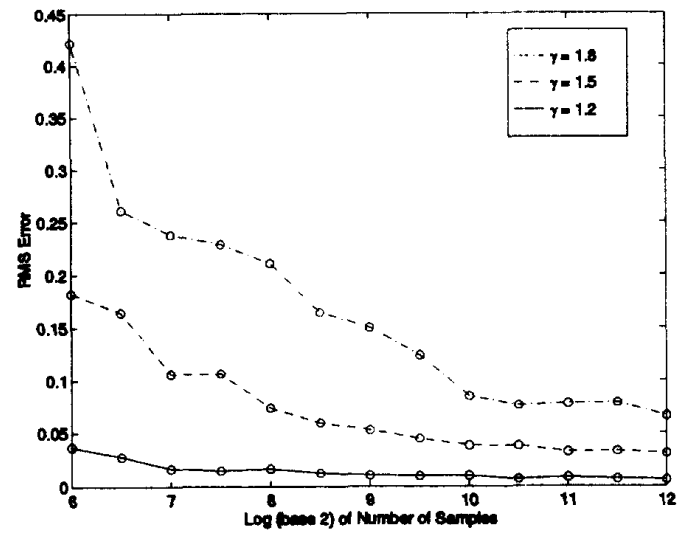

Figure 2: Dependence of parameter estimator performance on $N$ and $\gamma ; \rho=2, \alpha=1 / 15$

provided $\hat{\lambda}_{m} \neq \hat{\alpha}$; otherwise,

$$
E[x[n] \mid y[n], m ; \hat{\Theta}]=y[n] / 2 \text {. }
$$

Straightforward variants of this algorithm apply when some of the parameters are known a priori. In particular, parameter estimates of any known parameters in the algorithm are replaced with their true values in both the $\mathrm{E}$ - and $\mathrm{M}$-steps, and the corresponding parameter update in the M-step is omitted. In all cases, the EM algorithm increases the likelihood function at each iteration and converges to the ML estimates. Not surprisingly, however, the convergence rate of the algorithm generally improves when some of the parameter values are known.

In some preliminary experiments, the dyadic version $(\rho=2)$ of this algorithm was tested on simulated data. While all three parameters $\lambda, \alpha, \beta$ were assumed unknown and were estimated throughout the experiments, we focus on the performance of estimates for $\beta$ (and, hence, $\gamma$ ) since this is the primary parameter of interest.

In one set of experiments, we investigated the estimator performance as a function of sample size $N$ and $\gamma$, with the noise parameter fixed at $\alpha=15^{-1}$. To ensure that modeling error effects were included in these tests, both the power-law random variables and exponential noise terms in the test data were synthesized via transformation of uniform random variables. The results of the experiments are shown in Fig. 2. The RMS errors of the $\gamma$ estimates were taken over 64 Monte Carlo trials. As we would expect, better estimates are obtained when larger data sets are available. The apparent relationship between performance and the shape parameter $\gamma$ can be understood as follows. When $\gamma \approx 1$, extremely long and extremely short interarrivals occur with comparable frequency. In this case, very few data points are sufficient to capture the behavior of the probability density function over a broad range. For $\gamma \approx 2$, however, short interarrivals predominate over longer ones. Hence, a narrower range of the distribution is generally oberved, making it difficult to accurately estimate $\gamma$.

In a separate set of experiments, the effects of the quantity $\alpha / \lambda$ were examined, and the corresponding results are

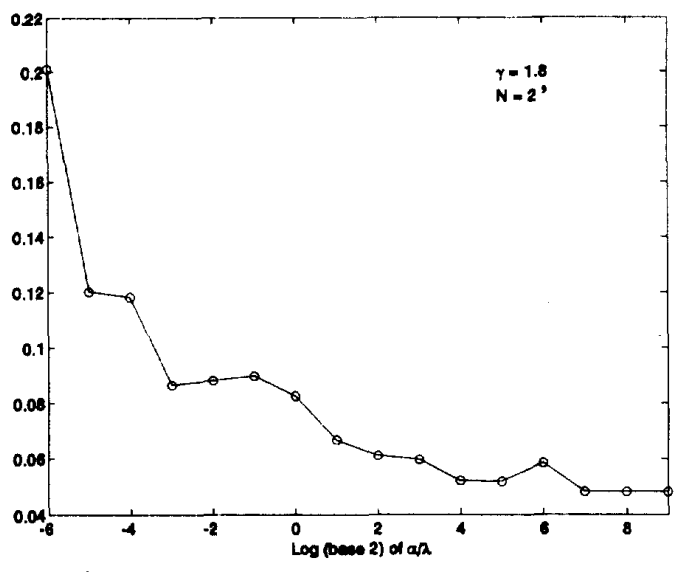

Figure 3: Dependence of parameter estimator performance on $\alpha / \lambda ; \rho=2$

shown in Fig. 3. As before, the estimator variance was taken from 64 trials. Since large values of $\alpha / \lambda$ correspond to low distortion in observations, the performance of the estimator improves as this quantity increases. Note that to allow specification of the true value of $\lambda$, the synthesis of the power-law random variables was based on the multiscale framework in this case.

These results, together with the results of a more extensive evaluation of this algorithm contained in [6], suggest that these multiscale estimation algorithms are robust, computationally efficient, and highly practical for a variety of applications.

\section{REFERENCES}

[1] B. B. Mandelbrot, The Fractal Geometry of Nature. San Francisco, CA: Freeman, 1982.

[2] B. B. Mandelbrot, "Self-similar error clusters in communication systems and the concept of conditional stationarity," IEEE Trans. Commun. Technol., vol. COM-13, pp. 71-90, Mar. 1965.

[3] M. C. Teich, "Fractal character of the auditory neural spike train," IEEE Trans. Biomed. Eng., pp. 150-160, Jan. 1989.

[4] M. Schroeder, Fractals, Chaos, Power Laws. New York, NY: W. H. Freeman, 1991.

[5] G. W. Wornell, "Wavelet-based representations for the $1 / f$ family of fractal processes," Proc. IEEE, vol. 81, pp. 1428-1450, Oct. 1993.

[6] W. M. Lam and G. W. Wornell, "Multiscale representations for a class of fractal point processes," IEEE Trans. Signal Processing, June 1994. Submitted for publication.

[7] D. H. Johnson and A. R. Kumar, "Modeling and analyzing fractal point processes," in Proc. Int. Conf. Acoust. Speech, Signal Processing, 1990.

[8] N. M. Laird, A. P. Dempster, and D. B. Rubin, "Maximum likelihood from incomplete data via the EM algorithm," Ann. Roy. Stat. Soc., pp. 1-38, Dec. 1977. 